\title{
Cardiac Tamponade in a Patient with Primary Hypothyroidism
}

\author{
${ }^{1}$ R Katyayini, ${ }^{2} \mathrm{NH}$ Rekha, ${ }^{3} \mathrm{R}$ Chikkananjaiah
}

\begin{abstract}
Hypothyroidism is a common disease with multisystem involvement. It may present clinically in various forms. Pericardial effusion (PE) is one of the cardiovascular manifestation.

Pericardial effusion is the accumulation of fluid in the pericardial space. The occurrence of pericardial effusion in hypothyroidism appears to be frequent manifestation of severe myxedema, than in mild hypothyroidism. The incidence of pericardial effusion in hypothyroidism is 3 to $6 \%$. However, the incidence of cardiac tamponade in patients with hypothyroidism is rare event. The slow accumulation of liquid in the pericardial space leads to the rarity of hemodynamic premonitory signs, even in presence of large effusions.

In this article, we report an elderly female presented with features of hypothyroidism and pericardial effusion which rapidly developed into cardiac tamponade. She did not have premonitory hemodynamic signs.
\end{abstract}

Keywords: Cardiac tamponade, Myxedema, Pericardial effusion.

How to cite this article: Katyayini R, Rekha $\mathrm{NH}$, Chikkananjaiah R. Cardiac Tamponade in a Patient with Primary Hypothyroidism. J Med Sci 2015;1(1):7-9.

\section{Source of support: Nil}

Conflict of interest: None

\section{INTRODUCTION}

Hypothyroidism is a disease with multisystem involvement which may present in various forms. Pericardial effusion is one of the cardiac manifestations in myxedema. ${ }^{1}$ Cardiac tamponade is usually a consequence of increased pericardial pressure with accumulation of fluid in the pericardial space. Cardiac tamponade as a complication of hypothyroidism is very rare. ${ }^{2}$ This low incidence is most likely due to slow accumulation of fluid and gradual pericardial distention. Hypothyroidism is characterized by low metabolic demands and, therefore, despite of depressed cardiac contractility and cardiac output,

\footnotetext{
${ }^{1}$ Postgraduate Student, ${ }^{2}$ Associate Professor, ${ }^{3}$ Professor

${ }^{1-3}$ Department of General Medicine, RajaRajeswari Medical College \& Hospital, Bengaluru, Karnataka, India
}

Corresponding Author: NH Rekha, Associate Professor Department of General Medicine, No. 128, 3rd Cross 2nd Main Ideal Homes, RajaRajeswari Nagar, Bengaluru, Karnataka India, e-mail: drrekhanh@gmail.com cardiac function remains sufficient to sustain the workload imposed on the heart. Another feature that distinguishes cardiac tamponade caused by hypothyroidism from other causes of tamponade is the absence of sinus tachycardia as a mechanism to maintain cardiac output. ${ }^{3}$

\section{CASE REPORT}

We report a case of 50 years old female presented with 2 months history of progressive swelling of lower limbs associated with puffiness of face, generalized weakness, change in voice, slowness of speech, lethargy and progressive weight gain. She was not diabetic. A patient was diagnosed to have hypertension few months before and was on treatment. At admission, her vital parameters were normal, blood pressure was 160/110 mm Hg, jugular venous pressure was not rised, and BMI was $32 \mathrm{~kg} / \mathrm{m}^{2}$. Her physical examination revealed periorbital puffiness, had loss of hair on lateral 1/3rd of eyebrow, coarse dry skin, nonpitting type of pedal edema of extremities and hoarseness of voice. Cardiac examination revealed muffled heart sounds. Patient had delayed relaxation of ankle jerk. Patient was clinically diagnosed for hypothyroidism and was investigated.

Her complete hemogram, renal function tests, blood sugar levels, liver function tests were normal. Lipid profile showed elevated triglycerides and total cholesterol. Electrocardiogram showed low voltage complexes with sinus bradycardia. Thyroid functions showed TSH $>150$ units, T4-1.386 ng/dl, T3 - 45 $\mu \mathrm{g} / \mathrm{dl}$. Creatine phospho kinase (CPK) and lactate dehydrogenase ( $\mathrm{LDH})$ were elevated. Two-dimensional (2D) echo at admission showed mild pericardial effusion (PE), with normal ejection fraction. Chest X-ray showed mild cardiomegaly. Echocardiogram revealed mild pericardial effusion (Fig. 1).

Patient was started on tablet thyronorm $75 \mu \mathrm{g}$ (thyroxine) once a day. On day 4 of hospitalization, patient complained of heaviness in the chest, however, there were no additional clinical findings on day 4 . Patient was taken for repeat echo which showed moderate pericardial effusion, early signs of pericardial tamponade, right ventricle (RV) and right atrial (RA) diastolic collapse, ejection fraction was normal, mild pulmonary artery hypertension (PAH), pulmonary artery systolic pressure (PASP) $35 \mathrm{~mm} \mathrm{Hg}$ (Fig. 2). Even with above findings, patient did not have 

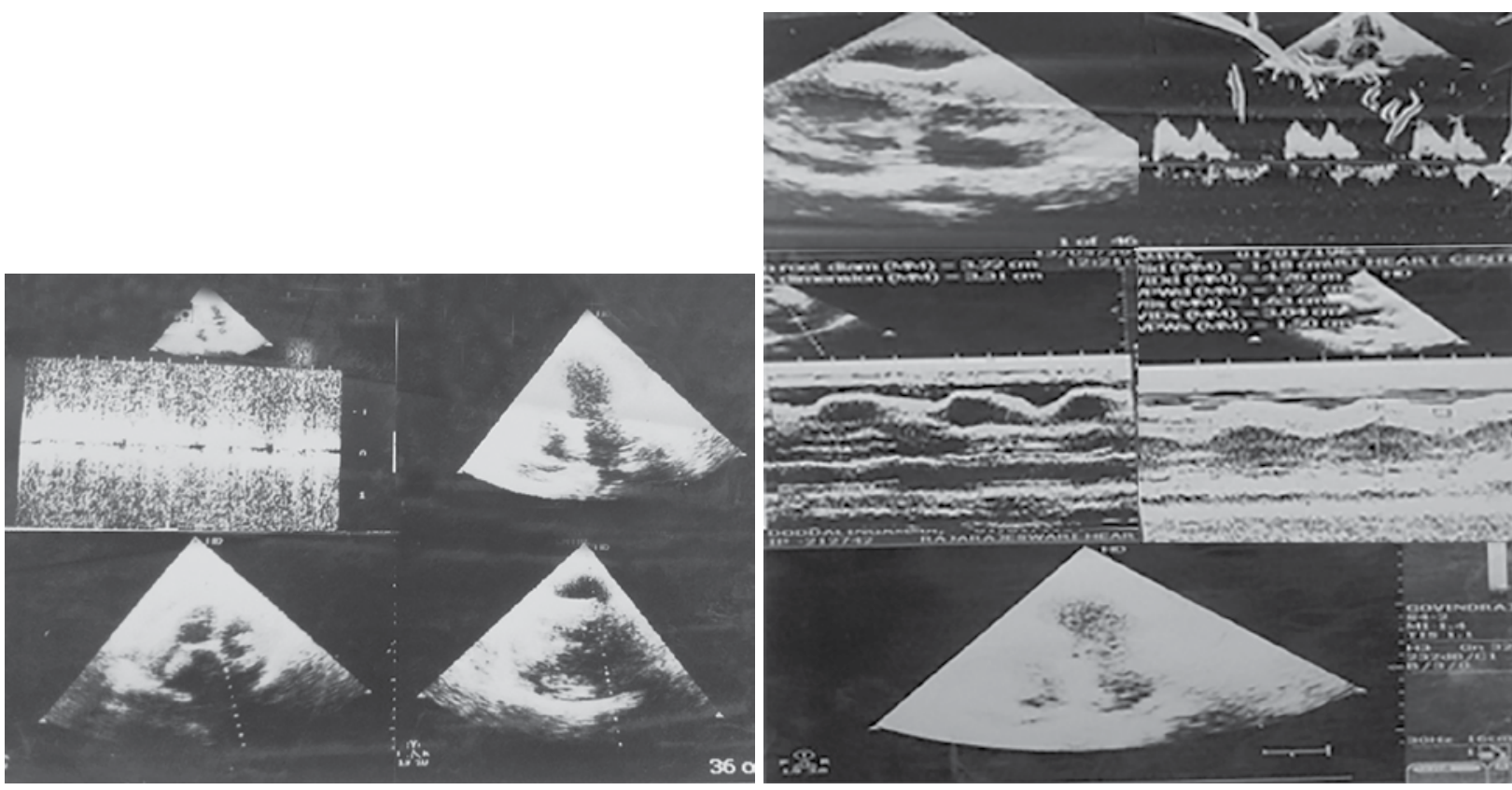

Fig. 1: Two-dimensional echo at admission with features of mild pericardial effusion
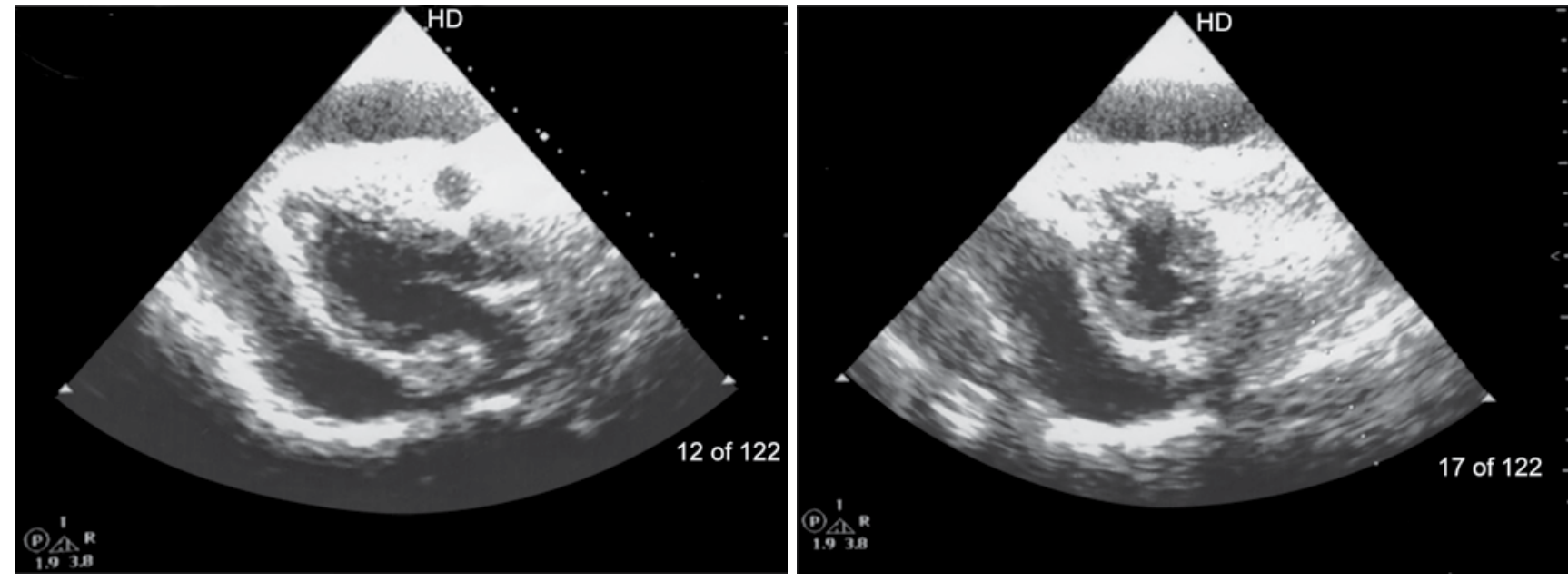

Fig. 2: Two-dimensional echo after 4 days of admission with findings of cardiac tamponade

hypotension, jugular venous pressure was normal, and patient was continued on medical management.

On day 7, 2D echo was repeated; however, findings of pericardial tamponade were same, patient was seen by a cardiologist, and medical management was advised. Patient was discharged with tab thyronorm $75 \mu \mathrm{g}$ per day.

Patient was continued on tab thyronorm $75 \mu \mathrm{g}$ and asked to follow-up after 4 weeks for repeat thyroid function tests.

Patient was reassessed by echocardiography after 4 weeks of thyroxine hormone treatment. There was complete disappearance of echocardiographic findings of PE and cardiac tamponade (Fig. 3).

\section{DISCUSSION}

The first known PE in hypothyroid patient dates back to 1918.

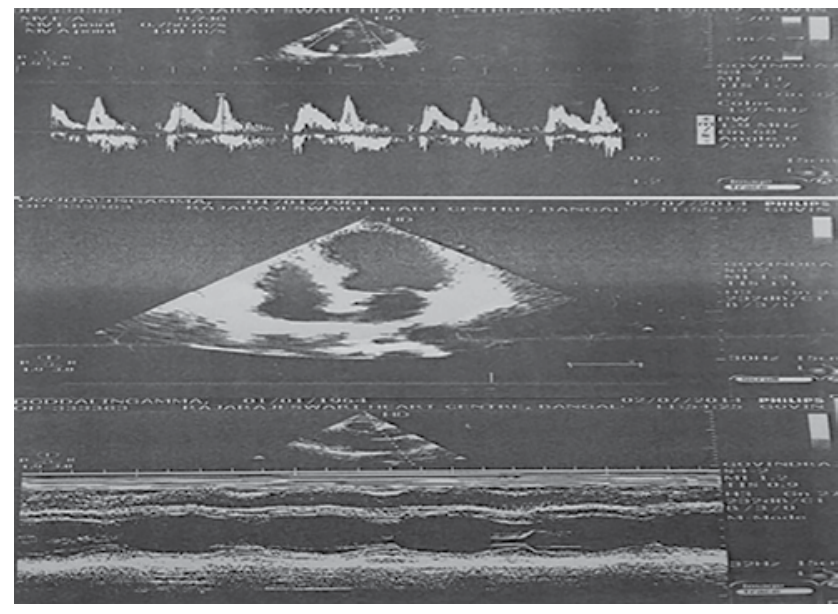

Fig. 3: Normal 2D echo after 4 weeks of thyroxine supplementation

Since then, there are several publications have reported on the association between hypothyroidism and pericardial effusion and even other serous effusion. ${ }^{4}$ 
The recent studies concluded that $\mathrm{PE}$ is infrequent in hypothyroidism with incidence of 3 to $6 \%{ }^{2}$ The pathophysiologic derangements responsible for the collection of fluid in the serous cavities of hypothyroid patients are probably increased systemic capillary permeability and disturbances in electrolyte metabolism. ${ }^{5}$ There is also evidence of greater than normal proportion of exchangeable albumin is localized to extravascular space due to decrease in albumin degradation. Alexander first used term 'gold paint effusion' to describe the golden-brown appearance of the pericardial fluid due to shimmering cholesterol crystals. The high cholesterol content of fluid has been attributed to disturbances in lipid metabolism. A churning action of the heart play a role in the precipitation of cholesterol from pericardial fluid and also poor absorptive capacity of pericardium may be a another major factor. ${ }^{5}$ Thus, PE may be frequent manifestation in myxedema in advanced severe stage but rare in early stage of hypothyroidism. ${ }^{2}$

Identification of cardiac tamponade in hypothyroidism is difficult and commonly mistaken for cardiac failure due to its symptoms and signs. Cardiac tamponade as complication of hypothyroidism is very rare. This low incidence is probably due to the slow accumulation of fluid and cardiac distensibility. Cardiac tamponade in hypothyroidism is mainly diagnosed by excluding other causes, like malignancy, tuberculosis, which are most frequent causes of nontraumatic PE with tamponade. ${ }^{6}$

Factors provoking cardiac tamponade include infection, spontaneous pericardial hemorrhage, thyroid hormone therapy. ${ }^{6}$ The mainstay of treatment for hypothyroid with $\mathrm{PE}$ is to simple thyroid hormone replacement. However, those patients with pericardial tamponade or impending tamponade as the patients develop paradoxical tachycardia as a warning sign for tamponade, the condition mandates urgent pericardiocentesis. How rapidly pericardial fluid gets accumulated is more important as per cardiac hemodynamics is concerned rather than the volume of effusion.

\section{CONCLUSION}

Hypothyroid with cardiac tamponade is one of the rare life-threatening condition. Bedside echocardiography is one of the gold standard investigation to diagnose PE with cardiac tamponade. Primary hypothyroidism with PE rapidly evolving into cardiac tamponade is unusal clinical presentation. Our patient presented with severe hypothyroidism with PE initially at admission and rapidly developing features of cardiac tamponade on echocardiography after initiating thyroxine supplementation. However, our patient did not have clinical signs of cardiac tamponade. Hence, patient was managed conservatively with hormonal supplementation. Our patient responded to medical management. Satisfactory clinical resolution of $\mathrm{PE}$ is usual outcome with thyro-xine replacement. Myxedema patients with PE should be carefully followed-up for an extended period, even when the patient has been successfully treated with thyroxine replacement and rendered euthyroid.

\section{REFERENCES}

1. Rachid A, Caum LC, Trentini AP, Fischer CA, Antonelli DAJ, Hagemann RP. Pericardial effusion with cardiac tamponade as a form of presentation of primary hypothyroidism. Arq Bras Cardiol 2002;78(6):583-585.

2. Kabadi UM, Kumar SP. Pericardial effusion in primary hypothyroidism. Am Heart J 1990 Dec;120(6 pt 1):1393-1395.

3. Parving HH, Hansen JM, Nielsen SL, Rossing N, Munck $\mathrm{O}$, Lassen NA. Mechanisms of edema formation in myxedema-increased protein extravasation and relatively slow lymphatic drainage. N Engl J Med 1979 Aug 30;301(9): 460-465.

4. Gottehrer A, Roa J, Stanford GG, Chernow B, Sahn SA. Hypothyroidism and pleural effusions. Chest 1990 Nov; 98(5):1130-1132.

5. Chou SL, Chern CH, How CK, Wang LM, Huang CI, Lee $\mathrm{CH}$. A rare case of massive pericardial effusion secondary to hypothyroidism. J Emerg Med 2005 Apr;28(3): 293-296.

6. Smolar EN, Rubin JE, Avramides A, Carter AC. Cardiac tamponade in primary myxedema and review of the literature. Am J Med Sci 1976 Nov-Dec;272(3):345-352. 